\title{
Lipopolysaccharide-induced tumor necrosis factor- $\alpha$ factor enhances inflammation and is associated with cancer (Review)
}

\author{
JUNRONG ZOU, PEI GUO, NONGHUA LV and DEQIANG HUANG \\ Research Institute of Digestive Diseases, The First Affiliated Hospital of Nanchang University, \\ Nanchang, Jiangxi 330006, P.R. China
}

Received September 13, 2014; Accepted June 3, 2015

DOI: $10.3892 / \mathrm{mmr} .2015 .4243$

\begin{abstract}
Lipopolysaccharide-induced tumor necrosis factor- $\alpha$ factor (LITAF) exerts transcription factor activity and is involved in protein quality control. LITAF activity is highly dependent on correct translocation from the endosome/lysosome to the nucleus, while certain LITAF mutants mislocalize to areas, such as the cytosol and mitochondria, resulting in developmental diseases. In addition, previous studies have proposed that LITAF functions as a tumor suppressor and is frequently under-represented in certain types of cancer. However, the mechanism of this phenomenon remains unclear. The present review summarizes the major advances in LITAF studies, and proposes that LITAF may serve as a switch in the balance between classical and alternative activation in tumor associated-inflammation. Thus, LITAF may be a promising therapeutic target with regard to the tumor microenvironment.
\end{abstract}

\section{Contents}

1. Introduction

2. Structure and general features of LITAF

3. Trafficking of LITAF

4. LITAF and STAT6 (B) in inflammation

5. LITAF and inflammatory diseases

6. LITAF and cancer

7. Summary and prospect

Correspondence to: Professor Deqiang Huang, Research Institute of Digestive Diseases, The First Affiliated Hospital of Nanchang University, 17 Yongwaizen Street, Nanchang, Jiangxi 330006, P.R. China

E-mail: hdq0515@163.com

Key words: lipopolysaccharide-induced tumor necrosis factor- $\alpha$ factor, inflammation, tumor

\section{Introduction}

As first identified by Polyak et al in 1997 (1), the gene of lipopolysaccharide (LPS)-induced tumor necrosis factor (TNF)- $\alpha$ factor (LITAF) was initially termed $p 53$-inducible gene 7 (PIG7), due to the fact that it encodes for a protein that is positively regulated by the tumor suppressor protein, p53 (1). Two years later, Myokai et al (2) cloned an LPS-regulated gene with the same sequence as PIG7. This gene was subsequently termed LITAF as its encoded protein product, LITAF, translocated into the nucleus following cellular activation by LPS, which was followed by the upregulation of $T N F-\alpha$ transcription (2-4).

It is widely accepted that tumor-associated inflammation is a major contributor to cancer progression, and it has been recognized as the seventh hallmark of cancer $(5,6)$. Numerous primary inflammatory mediators have been identified, including interleukin (IL)-4 (7), CCL18 (8) and granulocyte macrophage colony-stimulating factor (9). Previous observations suggest that LITAF, as a ubiquitously expressed gene (1-4), may be an enhancer of inflammatory diseases, as well as a suppressor of cancer-associated inflammation. In the current review, the above-mentioned observations are summarized, and LITAF is presented as a potential novel target for cancer therapy.

\section{Structure and general features of LITAF}

Human LITAF is located on chromosome 16 and it encodes a full length cDNA of 1,551 base pairs (bp), which contain three major structural components: A 5' untranslated region (UTR) of 1,001 bp, 3' UTR of $76 \mathrm{bp}$ and an open reading frame of $474 \mathrm{bp}(2,10)$. The C-terminal of the LITAF protein has enriched cysteine residues and includes a highly conserved $\mathrm{C} 3 \mathrm{H} 4$ zinc finger region that is interrupted by 23 hydrophobic amino acids, called small integral membrane protein of lysosome/late endosome (SIMPLE)-like domain (SLD) (11). The SLD domain contains a YXX $\varnothing$ ( $\varnothing$ is a hydrophobic amino acid) and double leucine motifs (12). It was reported that proteins containing the YXX $\varnothing$ motif interact with the clathrin adaptor compound and are, therefore, able to mediate the import and export of membrane proteins in the endosome, Golgi apparatus and lysosomes $(13,14)$. Furthermore, proteins with double leucine motifs are able to target lysosomes and endosomes (15). However, 


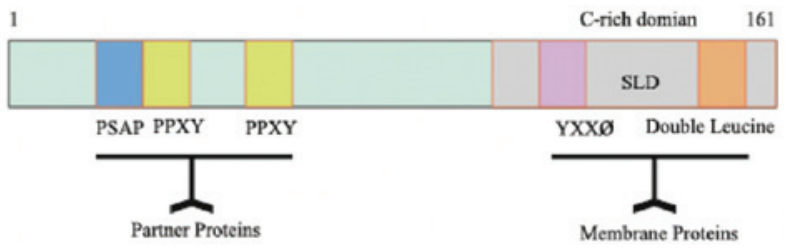

Figure 1. Structure of lipopolysaccharide-induced tumor necrosis factor- $\alpha$ factor.

the N-terminal of the LITAF protein is enriched with proline residues and has PPXY and PS/TAP motifs, which mediate the association of LITAF with partner proteins (16-18) (Fig. 1).

\section{Trafficking of LITAF}

The nuclear translocation and transcription factor activity of LITAF are critical for the activation of numerous immune cells via classical pathways (Fig. 2). While intracellular LITAF is located in the membranes of late endosomes and lysosomes under quiescent conditions, these processes require free LITAF to be released from these intracellular compartments. It has been proposed that such a process is orchestrated by the protein-protein interactions with ubiquitination-associated proteins, such as the E3 ligase NEDD4 (16). LITAF functions with the endosomal sorting complex required for transport components to control endosome-to-lysosome trafficking (17). As a negative control, previous studies have indicated that mutated LITAF proteins mislocalize to the cytosol (18) and/or mitochondria (19), where they cease their wild-type (WT) activities and serve as an etiological cause of Charcot-Marie-Tooth disease, a severe peripheral nervous system disorder $(20,21)$.

\section{LITAF and STAT6 (B) in inflammation}

LITAF is known as a TNF- $\alpha$ inducer (22), therefore, it is notable that transient transfection of LITAF resulted in no significant elevation of TNF- $\alpha$ levels following LPS treatment (3). This indicates that LPS activates additional factors, other than LITAF, that also regulate the transcription of $T N F-\alpha$ and that these factors may be binding partners of LITAF. Using a yeast two-hybrid system, a transcription factor, signal transducer and activator of transcription (STAT)6 (B), has been identified as a functional binding partner of LITAF (3). LITAF and STAT6 (B) are activated by LPS, then associate with toll-like receptor-2/4 to form a complex, which is dependent on MyD88 and is phosphorylated by p38- $\alpha$ (3). Phosphorylated LITAF and STAT6 (B) consequently interact to form a protein complex prior to translocating into the nucleus, where LITAF binds specifically to the promoter sequence, thus activating the expression of downstream genes, such as $T N F-\alpha$ and $I L-6(4,23)$ (Fig. 3). Focusing on this pathway, LITAF has become a novel target for the treatment of endotoxic shock and inflammation (24), as implicated by Matsuno et al (25) who demonstrated that LITAF-knockout mice were more resistant to LPS-induced mortality.

\section{LITAF and inflammatory diseases}

As a significant disease associated with LITAF, inflammatory bowel disease (IBD) is a type of chronic intestinal inflammatory disease with an unknown etiology, which includes ulcerative colitis (UC) and Crohn's disease (CD) (26). The typical pathogenesis of IBD includes aberrant expression of bowel-specific proinflammatory cytokines, including TNF- $\alpha(24,27,28)$. This indicates that LITAF may be involved in IBD and may be abnormally expressed in this disease. Stucchi et al (29) observed that the mRNA levels of LITAF in colon tissue samples from patients with $\mathrm{CD}$ were five times higher than those from healthy controls. In addition, within the same CD sample, the inflammatory areas presented with $60 \%$ more LITAF mRNA than the non-inflammatory areas (29). Similar phenomena have been observed in patients with UC. Colon tissues from patients with UC expressed LITAF mRNA levels 15 times greater than healthy individuals (26). However, in such patients, there was no significant difference in the mRNA level of LITAF between the inflammatory areas and the surrounding normal tissues. Immunohistochemistry has demonstrated that LITAF is predominantly expressed by lamina propria macrophages (LPM) (29). This was verified by Bushell et al (30) with a 2,4,6-trinitrobenzene sulfonic acid (TNBS)-induced mouse colon inflammation model. This study additionally indicated that mRNA and protein levels of LITAF were dramatically upregulated in TNBS-treated mice when compared with untreated mice. Furthermore, the expression of $T N F-\alpha$ in the LPM from LITAF mac $^{-/}$mice was significantly lower than that of the WT mice (30). These results strongly suggest that LITAF upregulates expression of TNF- $\alpha$ in LPM and elevated expression of LITAF coincides with the progression of IBD.

Arthritis is an inflammatory disease occurring in the joints of the human body and surrounding tissues, which has a complex etiology. Causal factors include chronic inflammation, autoimmune reactions, infection, metabolic disorders, trauma and degenerative disorders (31). Patients with arthritis commonly exhibit vascular endothelial dysfunction with alterations in numerous inflammatory factors, including TNF- $\alpha$, IL-6 and IL-8 $(32,33)$. To investigate whether LITAF was involved in arthritis, Merrill et al (34) established an LITAF knockout mouse [tamLITAF(i) ${ }^{-1}$ ] through tamoxifen induction. LPS was used to treat WT and tamLITAF $(\mathrm{i})^{-/-}$mice and collagen-induced arthritis experiments were performed. The degree of disease severity was found to be dramatically higher in the WT mice than in the tamLITAF $(\mathrm{i})^{-/-}$mice, this observation was noted from 3 days post-treatment and the difference became more significant over time. In addition, pannus and synovitis inflammations were observed to be elevated in the tamLITAF(i $)^{-/-}$mice. Additionally, the degree of bone resorption was observed to be lower in tamLITAF $(\mathrm{i})^{-/-}$mice compared with the WT mice (34). These results suggest that in vivo depletion of LITAF effectively 


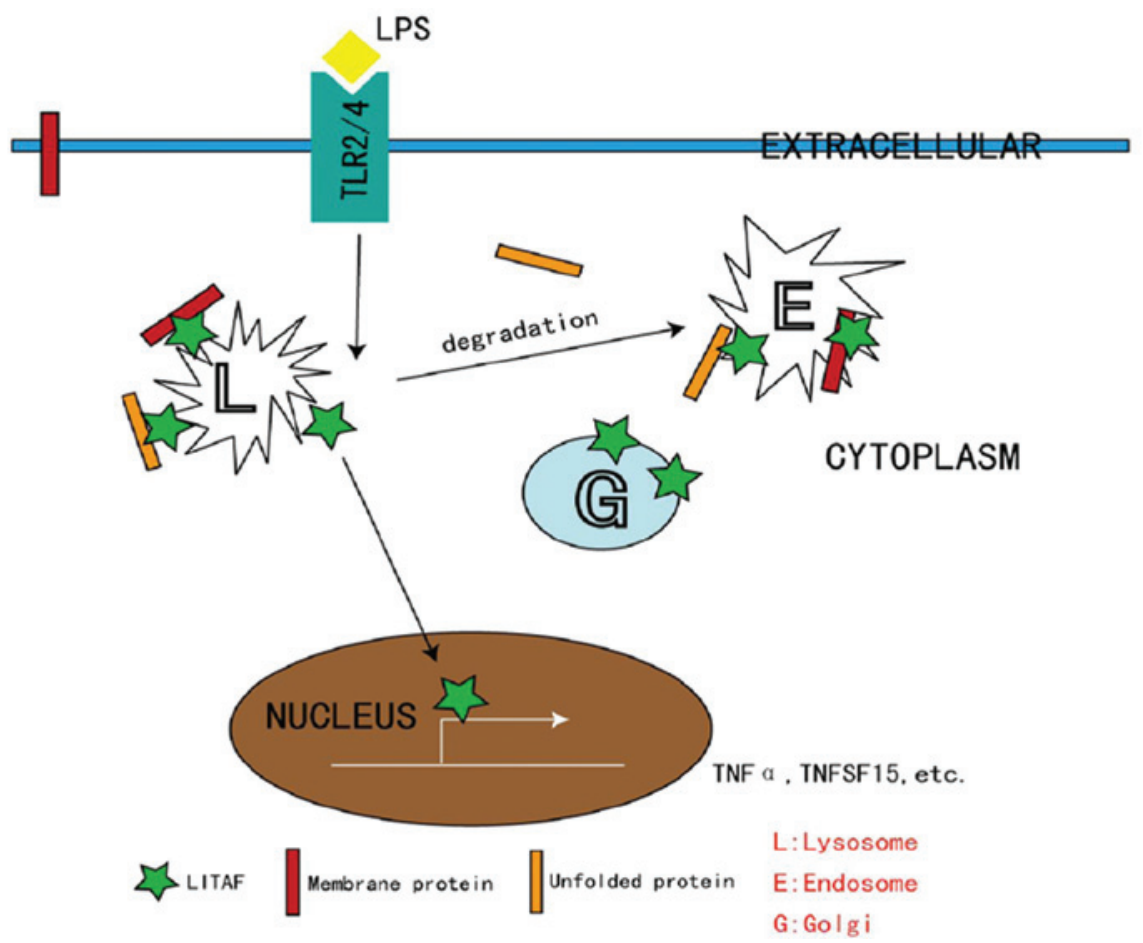

Figure 2. Schematic representation of subcellular localization of LITAF. LPS stimulation translocates LITAF from the cytoplasm into the nucleus to promote target gene expression. LITAF may target the lysosome or endosome to regulate protein degradation. LITAF, lipopolysaccharide-induced tumor necrosis factor- $\alpha$ factor; LPS, lipopolysaccharide; TLR2/4; toll-like receptor 2/4; TNF, tumor necrosis factor.

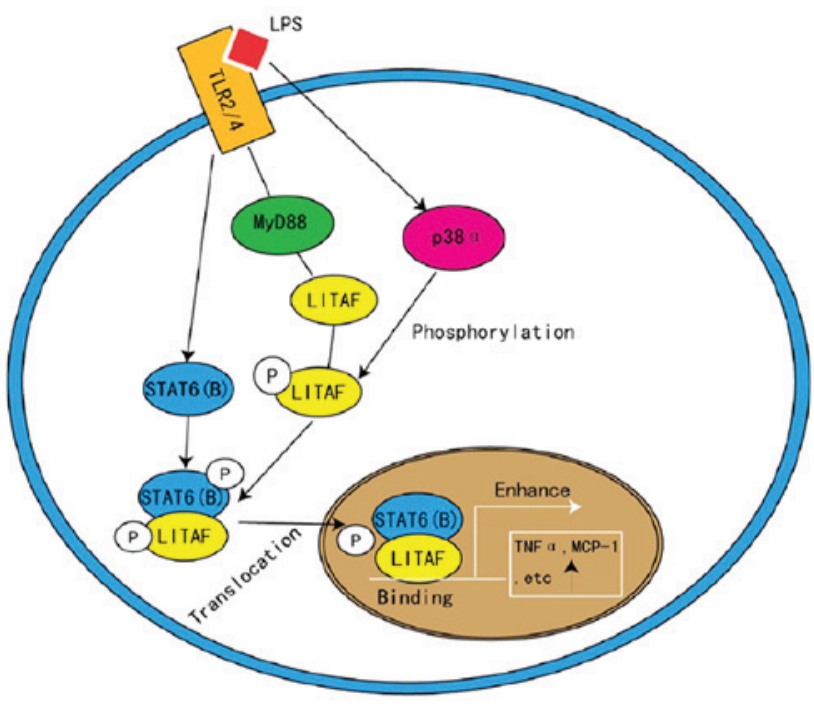

Figure 3. Mechanisms of inflammation regulation by LITAF. LITAF and its functional partner, STAT6 (B) can be activated by LPS/TLR2/4 signaling, which involves phosphorylated $\mathrm{p} 38-\alpha$. Phosphorylated LITAF binds to STAT6 (B), which is followed by translocation into the nucleus and regulation of proinflammatory gene expression. LITAF, lipopolysaccharide-induced tumor necrosis factor- $\alpha$ factor; STAT6, signal transducer and activator of transcription 6; TLR2/4, toll-like receptor $2 / 4$.

reduces the harmful effects of arthritis. Corroborating these results, Srinivasan et al (35) identified a connection between LITAF and arthritis, and proposed that it may involve extracellular-related kinase $1 / 2$ and protein kinase B (35). These observations suggest that LITAF may promote the progression of arthritis, as well as additional associated whole body inflammation in mice.

\section{LITAF and cancer}

In addition to inflammation, LITAF has been identified as a potential tumor suppressor gene, due to the fact that its expression can be induced by p53 (1). Evidence from cohort studies has revealed that LITAF expression is significantly lower in tumor tissues when compared with isogenic normal tissues $(36,37)$. However, the functional mechanisms of the action of LITAF in tumors remains unclear.

Zhou et al (38) used small hairpin (sh)RNA to disrupt gene expression in the adenosine monophosphate-activated protein kinase (AMPK)-LITAF-TNF superfamily member 15 (TNFSF15) signaling pathway in prostatic cancer cells and elucidated that shRNA targeting of LITAF (shRNA-LITAF) significantly enhanced the degree of malignancy of cancer cells. Notably, its effect was more marked than that of shRNA-p53 (38). Furthermore, Zhou et al (38) established an allograft prostatic tumor model by subcutaneous injection of prostatic cancer cells into nude mice. Following development of tumors, those analyzed from the shRNA-LITAF group were observed to be significantly larger in size and weight compared with the tumors from the shRNA-control group (38). These results suggest that LITAF inhibits the proliferation of prostatic cancer cells, which supports the assumption that LITAF functions as a tumor suppressor gene.

Furthermore, a breast cancer study analyzed the gene expression of normal breast tissues, ductal carcinoma in situ (DCIS) and invasive ductal carcinoma (IDC) using the Serial analysis of gene expression method. The study revealed that LITAF expression was 29 times lower in DCIS compared with that of normal tissues, while there was no clear alteration in the LITAF levels observed in IDC (36). Similarly, 
Fernandez-Cobo et al (39) confirmed that LITAF expression in breast cancer cells was 37 times lower than that in normal breast epithelial cells. It was hypothesized that LITAF and other cytokines participate in the recovery process of breast tissues following pregnancy and lactation, during which extensive apoptotic events occur in breast tissues (40). Furthermore, lower expression of LITAF may promote the early transformation of breast tissues by slowing down the normal apoptotic process.

Wang et al (37) conducted qualitative polymerase chain reaction analysis and established that bone marrow LITAF expression in patients with acute leukemia (as well as refractory and relapsed acute leukemia) is significantly reduced, when compared with the expression levels in patients at initial diagnosis. In addition, Wang et al elucidated that the transient expression of LITAF has little apparent influence on the proliferation of acute leukemia cells. However, LITAF markedly enhances the inhibitory effects of etoposide and daunomycin on acute leukemia, suggesting that LITAF sensitizes leukemic cells to chemotherapeutic agents (37).

It should be noted that not all cancer cells exhibit low expression of LITAF. For example, Matsumura et al (41) examined a rare malignant skin tumor, extra-mammary Paget's disease (EMPD) and observed that EMPD tissues exhibited higher expression levels of LITAF in comparison with isogenic normal tissues, in three out of four individuals (41). This phenomenon may be relevant to somatic mutations. The study also identified LITAF site mutations in three out of 12 cases, among which two exhibited non-synonymous mutations and one exhibited synonymous mutations (41). The mechanism of this mutation and the associated expression remains unclear.

There are numerous mechanisms suggested to be involved in the tumor suppressor activity of LITAF (Fig. 4). Firstly, the two PPXY motifs at the N-terminal of LITAF associates with the WW domain containing proteins, such as NEDD4 and Itch, which are able to promote p53- and/or p72-mediated cell apoptosis and subsequently restrict tumor growth $(40,42,43)$. Secondly, LITAF may promote the ubiquitin-proteasome system in mediating the degradation of pro-cancerous proteins (44). Thirdly, LITAF is able to stimulate the expression of TNFSF15 and then restrain angiogenesis to inhibit tumor growth, as it acts as a downstream target of the tumor suppressor factor, AMPK (38).

It is hypothesized that LITAF may serve as a switch in the balance between classical inflammation and alternative activation in cancer. Immune cell infiltration is a typical trigger of cancer-associated inflammation. Notably, studies using mouse models suggested that the alleviation of immune responses results in a decline in the quantity and size of tumors in the murine body $(45,46)$. Alternative activation of various cell types, including tumor-associated macrophages (47), cancer-related fibroblasts (48) and aberrantly activated neutrophils (49) have been identified in numerous types of cancer, including breast (50) and colorectal cancer (51), and melanoma (52). In the context of these types of cancer, the regulators and determinants of classical and alternative immune activation remain unclear. It has been observed that LITAF is highly expressed in macrophages in various acute inflammatory tissues, and classically induces TNF- $\alpha$, which

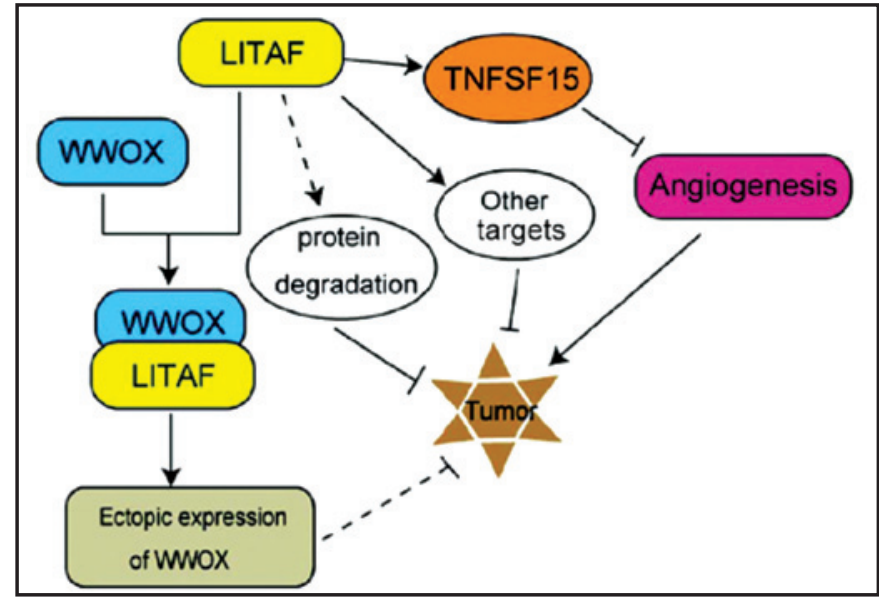

Figure 4. Possible mechanisms of the tumor suppressing role of LITAF LITAF, lipopolysaccharide-induced tumor necrosis factor- $\alpha$ factor.

exerts antiviral, antitumor and proapoptotic activities when at sufficiently high in situ concentrations $(53,54)$. Short-term activation of LITAF inhibits the growth of cancer cells potentially through proinflammatory effects that target the expansion of tumor-antigen specific $\mathrm{T}$ cells and the cancer cells themselves (55). During chronic inflammation, inflammatory factors overexpressed by the alternative activated immune cells may suppress the expression of LITAF via the negative feedback mechanism, for example via the nitric oxide pathway (56). However, the exact role of LITAF in the transition from inflammation to tumor suppression requires further investigation, which may elucidate the potential for LITAF manipulation to modulate early carcinogenesis and/or cancer progression.

\section{Summary and prospect}

LITAF may affect cellular functions by either acting as a transcription factor in mediating target gene expression, or by acting as a recruiting factor that targets partner proteins to the lysosome for degradation. Current evidence indicates that various possible mechanisms may explain the contribution of altered LITAF expression to the progression of diseases, such as inflammation or tumors: i) Cytokine levels are dysregulated; ii) p53-mediated cell apoptosis signaling is affected; iii) Protein degradation in the lysosome is interrupted. It is proposed that LITAF may serve as a switch in the balance of classical and alternative activation in the tumor microenvironment. It remains unclear whether LITAF is a cause or effect of tumor inflammation, thus it is an important focus for further investigation and may be a promising therapeutic target.

\section{Acknowledgements}

The current study was supported in part by grants from The National Science Foundation of China (grant nos. 81171952, 8127292, 31460304 and 81460374) and a grant from Jiangxi Provincial Department of Science and Technology (grant no. 20133BBG70061). The authors would also like to thank Dr Zhijun Luo and Dr Yong Xie for their support. 


\section{References}

1. Polyak K, Xia Y, Zweier JL, Kinzler KW and Vogelstein B: A model for p53-induced apoptosis. Nature 389: 300-305, 1997.

2. Myokai F, Takashiba S, Lebo R and Amar S: A novel lipopolysaccharide-induced transcription factor regulating tumor necrosis factor alpha gene expression: Molecular cloning, sequencing, characterization and chromosomal assignment. Proc Natl Acad Sci USA 96: 4518-4523, 1999.

3. Tang X, Marciano DL, Leeman SE and Amar S: LPS induces the interaction of a transcription factor, LPS-induced TNF-alpha factor and STAT6 (B) with effects on multiple cytokines. Proc Natl Acad Sci USA 102: 5132-5137, 2005.

4. Tang X, Metzger D, Leeman S and Amar S: LPS-induced TNF-alpha factor (LITAF)-deficient mice express reduced LPS-induced cytokine: Evidence for LITAF-dependent LPS signaling pathways. Proc Natl Acad Sci USA 103: 13777-13782, 2006.

5. Coussens LM and Werb Z: Inflammation and cancer. Nature 420: 860-867, 2002.

6. Mantovani A: Cancer: Inflaming metastasis. Nature 457: 36-37, 2009.

7. Gocheva V, Wang H-W, Gadea BB, Shree T, Hunter KE, Garfall AL, Berman T and Joyce JA: IL-4 induces cathepsin protease activity in tumor-associated macrophages to promote cancer growth and invasion. Genes Dev 24: 241-255, 2010.

8. Chen J, Yao Y, Gong C, Yu F, Su S, Chen J, Liu B, Deng H, Wang F, Lin L, et al: CCL18 from tumor-associated macrophages promotes breast cancer metastasis via PITPNM3. Cancer Cell 19: 541-555, 2011.

9. Su S, Liu Q, Chen J, Chen J, Chen F, He C, Huang D, Wu W, Lin L, Huang W, et al: A Positive Feedback Loop between mesenchymal-like cancer cells and macrophages is essential to breast cancer metastasis. Cancer cell 25: 605-620, 2014.

10. Bolcato-Bellemin AL, Mattei MG, Fenton $\mathrm{M}$ and Amar S Molecular cloning and characterization of mouse LITAF cDNA role in the regulation of tumor necrosis factor-alpha (TNF-alpha) gene expression. J Endotoxin Res 10: 15-23, 2004.

11. Moriwaki Y, Begum NA, Kobayashi M, Matsumoto M Toyoshima K and Seya T. Mycobacterium bovis Bacillus Calmette-Guerin and its cell wall complex induce a nove lysosomal membrane protein, SIMPLE, that bridges the missing link between lipopolysaccharide and p53-inducible gene, LITAF(PIG7), and estrogen-inducible gene, EET-1. J Biol Chem 276, 23065-23076, 2001.

12. Boge M, Wyss S, Bonifacino JS and Thali M: A membrane-proximal tyrosine-based signal mediates internalization of the HIV-1 envelope glycoprotein via interaction with the AP-2 clathrin adaptor. J Biol Chem 273: 15773-15778, 1998.

13. Bonifacino JS and Dell'Angelica EC. Molecular bases for the recognition of tyrosine-based sorting signals. J Cell Biol 145 : 923-926, 1999.

14. Simmen T, Schmidt A, Hunziker W and Beermann F: The tyrosinase tail mediates sorting to the lysosomal compartment in MDCK cells via a di-leucine and a tyrosine-based signal. J Cell Sci 112: 45-53, 1999.

15. Letourneur F and Klausner RD: A novel di-leucine motif and a tyrosine-based motif independently mediate lysosomal targeting and endocytosis of CD3 chains. Cell 69: 1143-1157, 1992.

16. Shirk AJ, Anderson SK, Hashemi SH, Chance PF and Bennett CL: SIMPLE interacts with NEDD4 and TSG101: Evidence for a role in lysosomal sorting and implications for Charcot-Marie-Tooth disease. J Neurosci Res 82: 43-50, 2005.

17. Lee SM, Chin LS and Li L: Charcot-Marie-Tooth disease-linked protein SIMPLE functions with the ESCRT machinery in endosomal trafficking. J Cell Biol 199: 799-816, 2012.

18. Lee SM, Olzmann JA, Chin LS and Li L: Mutations associated with Charcot-Marie-Tooth disease cause SIMPLE protein mislocalization and degradation by the proteasome and aggresome-autophagy pathways. J Cell Sci 124: 3319-3331, 2011.

19. Ferreira Lacerda AF, Hartjes E and Brunetti CR: LITAF mutations associated with Charcot-Marie-Tooth Disease 1C Show mislocalization from the late endosome/lysosome to the mitochondria. PLoS One 9: e103454, 2014.

20. Ciotti P, Luigetti M, Geroldi A, Capponi S, Pezzini I, Gulli R, Pazzaglia C, Padua L, Massa R, Mandich P, et al: A novel LITAF/SIMPLE mutation within a family with a demyelinating form of Charcot-Marie-Tooth disease. J Neurol Sci 343: 183-186, 2014.
21. Luigetti M, Fabrizi GM, Taioli F, Del Grande A and Lo Monaco M: A novel LITAF/SIMPLE variant within a family with minimal demyelinating Charcot-Marie-Tooth disease. Neurol Sci 35: 2014

22. Tang X, Molina M and Amar S: p53 short peptide (p53pep164) regulates lipopolysaccharide-induced tumor necrosis factor-alpha factor/cytokine expression. Cancer Res 67: 1308-1316, 2007.

23. Tang $X$, Woodward $\mathrm{T}$ and Amar S: A PTP4A3 peptide PIMAP39 modulates TNF-alpha levels and endotoxic shock. J Innate Immun 2: 43-55, 2010.

24. Brannigan AE, Watson RW, Beddy D, Hurley H, Fitzpatrick JM and O'Connell PR: Increased adhesion molecule expression in serosal fibroblasts isolated from patients with inflammatory bowel disease is secondary to inflammation. Ann Surg 235: 507-511, 2002.

25. Matsuno H, Yudoh K, Katayama R, Nakazawa F, Uzuki M, Sawai T, Yonezawa T, Saeki Y, Panayi GS, Pitzalis C, et al: The role of TNF-alpha in the pathogenesis of inflammation and joint destruction in rheumatoid arthritis (RA): A study using a human RA/SCID mouse chimera. Rheumatology (Oxford) 41: 329-337, 2002

26. Stucchi A, Reed K, O'Brien M, Cerda S, Andrews C, Gower A, Bushell K, Amar S, Leeman S and Becker J: A new transcription factor that regulates TNF-alpha gene expression, LITAF, is increased in intestinal tissues from patients with CD and UC. Inflamm Bowel Dis 12, 581-587 2006.

27. Baker DA, Barth J, Chang R, Obeid LM and Gilkeson GS: Genetic sphingosine kinase 1 deficiency significantly decreases synovial inflammation and joint erosions in murine TNF-alpha-induced arthritis. J Immunol 185: 2570-2579, 2010.

28. Bushell KN, Leeman SE, Gillespie E, Gower AC, Reed KL, Stucchi AF, Becker JM and Amar S: LITAF mediation of increased TNF- $\alpha$ secretion from inflamed colonic lamina propria macrophages. PLoS One 6: e25849, 2011.

29. Stucchi A, Reed K, O'Brien M, Cerda S, Andrews C, Gower A, Bushell K, Amar S, Leeman S and Becker J: A new transcription factor that regulates TNF-alpha gene expression, LITAF, is increased in intestinal tissues from patients with CD and UC. Inflamm Bowel Dis 12: 581-587, 2006

30. Bushell KN, Leeman SE, Amar S, Reed KL, Gower AC, Stucchi AF and Becker JM: Macrophage-specific LITAF (lipopolysaccharide induced TNF-alpha factor) knockout mice (LITAF mac-/-) have a reduced inflammatory response to colonic administration of trinitrobenzene sulfonic acid (TNBS). FASEB J 22 (Meeting Abstract Supplement): 1138.4, 2008.

31. Zhang H, Hilton MJ, Anolik JH, Welle SL, Zhao C, Yao Z, Li X, Wang Z, Boyce BF and Xing L: NOTCH inhibits osteoblast formation in inflammatory arthritis via noncanonical NF- $\kappa \mathrm{B}$. J Clin Invest 124: 3200-3214, 2014

32. Feldmann M, Brennan FM and Maini RN: Role of cytokines in rheumatoid arthritis. Annu Rev Immunol 14: 397-440, 1996.

33. Brennan FM and McInnes IB: Evidence that cytokines play a role in rheumatoid arthritis. J Clin Invest 118: 3537-3545, 2008.

34. Merrill JC, You J, Constable C, Leeman SE and Amar S: Whole-body deletion of LPS-induced TNF- $\alpha$ factor (LITAF) markedly improves experimental endotoxic shock and inflammatory arthritis. Proc Natl Acad Sci USA 108: 21247-21252, 2011.

35. Srinivasan S, Leeman SE and Amar S: Beneficial dysregulation of the time course of inflammatory mediators in lipopolysaccharide-induced tumor necrosis factor alpha factor-deficient mice. Clin Vaccine Immunol 17: 699-704, 2010.

36. Abba MC, Drake JA, Hawkins KA, Hu Y, Sun H, Notcovich C Gaddis S, Sahin A, Baggerly K and Aldaz CM: Transcriptomic changes in human breast cancer progression as determined by serial analysis of gene expression. Breast Cancer Res 6: R499-R513, 2004

37. Wang D, Liu J, Tang K, Xu Z, Xiong X, Rao Q, Wang M and Wang J: Expression of pig7 gene in acute leukemia and its potential to modulate the chemosensitivity of leukemic cells. Leuk Res 33: 28-38, 2009.

38. Zhou J, Yang Z, Tsuji T, Gong J, Xie J, Chen C, Li W, Amar S and Luo Z: LITAF and TNFSF15, two downstream targets of AMPK, exert inhibitory effects on tumor growth. Oncogene 30: 1892-1900, 2011

39. Fernandez-Cobo M, Holland JF and Pogo BG: Transcription profiles of non-immortalized breast cancer cell lines. BMC Cancer 6: 99, 2006. 
40. Ludes-Meyers JH, Kil H, Bednarek AK, Drake J, Bedford MT and Aldaz CM: WWOX binds the specific proline-rich ligand PPXY: identification of candidate interacting proteins. Oncogene 23: 5049-5055, 2004.

41. Matsumura Y, Matsumura Y, Nishigori C, Horio T and Miyachi Y: PIG7/LITAF gene mutation and overexpression of its gene product in extramammary Paget's disease. Int J Cancer 111: 218-223, 2004.

42. Takeuchi T, Adachi Y and Nagayama T: A WWOX-binding molecule, transmembrane protein 207 , is related to the invasiveness of gastric signet-ring cell carcinoma. Carcinogenesis 33: 548-554, 2012.

43. Eaton HE, Metcalf J, Lacerda AF and Brunetti CR: Accumulation of endogenous LITAF in aggresomes. PLoS One 7: e30003, 2012

44. Eaton HE, Desrochers G, Drory SB, Metcalf J, Angers A and Brunetti CR: SIMPLE/LITAF expression induces the translocation of the ubiquitin ligase itch towards the lysosomal compartments. PLoS One 6: e16873, 2011.

45. van Kempen LC, de Visser KE and Coussens LM: Inflammation, proteases and cancer. Eur J Cancer 42: 728-734, 2006.

46. de Visser KE and Coussens LM: The inflammatory tumor microenvironment and its impact on cancer development. Contrib Microbiol 13: 118-137, 2006.

47. Mantovani A, Schioppa T, Porta C, Allavena P and Sica A: Role of tumor-associated macrophages in tumor progression and invasion. Cancer Metastasis Rev 25: 315-322, 2006.

48. Spaeth EL, Dembinski JL, Sasser AK, Watson K, Klopp A, Hall B, Andreeff $M$ and Marini F: Mesenchymal stem cell transition to tumor-associated fibroblasts contributes to fibrovascular network expansion and tumor progression. PloS one 4: e4992, 2009.
49. Galdiero MR, Garlanda C, Jaillon S, Marone G and Mantovani A: Tumor associated macrophages and neutrophils in tumor progression. J Cell Physiol 228: 1404-1412, 2013.

50. Qian B, Deng Y, Im JH, Muschel RJ, Zou Y, Li J, Lang RA and Pollard JW: A distinct macrophage population mediates metastatic breast cancer cell extravasation, establishment and growth. PLoS One 4: e6562, 2009.

51. Itzkowitz $\mathrm{SH}$ and $\mathrm{Y}$ io $\mathrm{X}$ : Inflammation and cancer IV. Colorectal cancer in inflammatory bowel disease: The role of inflammation. Am J Physiol Gastrointest Liver Physiol 287: G7-G17, 2004.

52. Gazzaniga S, Bravo AI, Guglielmotti A, van Rooijen N, Maschi F, Vecchi A, Mantovani A, Mordoh J and Wainstok R: Targeting tumor-associated macrophages and inhibition of MCP-1 reduce angiogenesis and tumor growth in a human melanoma xenograft. J Invest Dermatol 127: 2031-2041, 2007.

53. Bazzoni $\mathrm{F}$ and Beutler B: The tumor necrosis factor ligand and receptor families. N Engl J Med 334: 1717-1725, 1996.

54. Locksley RM, Killeen N and Lenardo MJ: The TNF and TNF receptor superfamilies: Integrating mammalian biology. Cell 104: 487-501, 2001.

55. Zhou J, Yang Z, Tsuji T, Gong J, Xie J, Chen C, Li W, Amar S and Luo Z: LITAF and TNFSF15, two downstream targets of AMPK, exert inhibitory effects on tumor growth. Oncogene 30: 1892-1900, 2011.

56. Pang T, Wang J, Benicky J and Saavedra JM: Minocycline ameliorates LPS-induced inflammation in human monocytes by novel mechanisms including LOX-1, Nur77 and LITAF inhibition. Biochim Biophys Acta 1820 503-510, 2012. 